\title{
GEOLOCALIZACIÓN. PONDERACIÓN DE LA SEGURIDAD PÚBLICA VS. PRIVACIDAD: ANÁLISIS DE LA ACCIÓN DE INCONSTITUCIONALIDAD 32/2012 DE LA SUPREMA CORTE DE JUSTICIA DE LA NACIÓN
}

\author{
Dayana SeVIlla Osornio*
}

\section{INTRODUCCIÓN}

Uno de los paradigmas que se ha instalado en la teoría y práctica jurídica es la solución de la colisión de principios a través de las llamadas teorías de la ponderación. La importancia de estas teorías radica en quienes las utilizan, ya que son los tribunales constitucionales quienes dirimen, en última instancia, en países de Latinoamérica ( $v$ g. México, Colombia, Argentina, Costa Rica, etcétera).

La ponderación constituye la medida para determinar los límites de los intereses legítimos. Ya en la jurisdicción administrativa, algunos países europeos antiformalistas (Alemania y Francia) habían propuesto formas de argumentar que podían considerarse muy cercanas a la idea de la ponderación (Sardo, 2013: 239-272). En algunos casos, el balanceo de los intereses era considerado también como la lógica que regulaba la aplicación de algunos institutos del derecho civil (por ejemplo, la disciplina de las inmisiones). Actualmente, y a pesar de sus detractores y críticos, Robert Alexy ha replanteado el juicio de proporcionalidad de manera analítica, lo cual se verá más adelante (Sardo, 2013).

En diciembre de 2011, en México, se llevó a cabo una de las mayores reformas constitucionales en materia de derechos humanos, a partir de la cual se reconoce su progresividad mediante la expresión clara del principio

* Licenciada en derecho por la Facultad de Derecho de la UNAM. Cuenta con estudios de posgrado en la Facultad de Derecho de la Universidad de Buenos Aires (UBA); dayana.sevilla@hotmail.com.

Fecha de recepción: 21 de enero de 2019.

Fecha de dictamen: 07 de abril de 2019. 
pro persona como rector de la interpretación y aplicación de las normas jurídicas en aquellas que favorezcan y brinden mayor protección a las personas en mira de la justiciabilidad y eficacia de los derechos. Lo anterior, a la postre, tiende al mejoramiento de las condiciones de vida de la sociedad $\mathrm{y}$ al desarrollo de cada persona en lo individual. ${ }^{1}$

La reforma también contempló dar mayores facultades a los tribunales para la interpretación en la aplicación de los principios expresados en la carta magna. Algunos tribunales, como los europeos y americanos, cuentan con una tradición más antigua sobre la argumentación en términos de proporcionalidad, lo cual ha dado lugar, en el ámbito académico, al planteamiento de distintos modelos de aplicación de la ponderación.

Un Estado constitucional de derecho se caracteriza por la incorporación de un conjunto de principios cuya estructura difiere de las reglas, en tanto no tienen definido el supuesto de hecho ni la obligación a imputar en caso de infracción. La presencia de esos principios representa un aumento de la discrecionalidad que, como potestad para determinar el sentido de los enunciados jurídicos, debe ser limitada como presupuesto básico del Estado de derecho, en el que toda expresión de poder debe estar condicionada por una norma jurídica (Estrada-Vélez, 2010: 77-112). En ese contexto tiene lugar la ponderación, asumida por un sector de la doctrina como un límite a la discrecionalidad, y por otro, como un ejercicio ilimitado de la misma. Optar por una u otra posición depende del reconocimiento de las transformaciones de la teoría del derecho en el contexto del Estado constitucional, y en especial de la noción de principios que se asuma. En síntesis, no es posible comprender la ponderación como método sin determinar de manera previa la naturaleza (jurídica o moral) del objeto sobre el cual se impone la ponderación.

La protección de datos personales es un tópico que ha tomado fuerza en los últimos años a través del desarrollo de un andamiaje jurídico especializado. En primer lugar, mediante la reforma constitucional al artículo 16, la cual estableció los derechos ARCO (acceso, rectificación, cancelación y oposición), buscando garantizar la protección de los datos personales en posesión de particulares y de sujetos obligados (entidades públicas). En segundo lugar, mediante leyes especializadas, como la Ley Federal de Protección de Datos Personales en Posesión de Particulares, la Ley General de Protección de Datos Personales en Posesión de Sujetos Obligados, y

\footnotetext{
1 Las reformas constitucionales en materia de amparo y derechos humanos, publicadas en junio de 2011, pueden consultarse en: http://www2.scjn.gob.mx/red/constitucion/ inicio.html.
} 
leyes locales en la materia. Además, se estableció a nivel constitucional un organismo garante en esta materia.

La geolocalización, al ser un dato personal sensible protegido por la normatividad antes señalada, debe ser regulado de manera cuidadosa en el marco del derecho de acceso a la información, ya que la injerencia indebida a esta información sensible puede causar un menoscabo en los derechos de las personas.

El uso de la geolocalización de una persona con fines de seguridad pública se vio excedida en su legislación ante modificadores normativos que dieron lugar a lo que se conoció como Ley de Geolocalización, con la cual se otorgaba la facultad discrecional al Ministerio Público para solicitar esta información a las empresas de telecomunicaciones con el fin de la persecución de delitos que cayeran en la hipótesis de delincuencia organizada, narcotráfico, entre otros calificados como graves. Esta legislación fue impugnada mediante una acción de inconstitucionalidad debido a la colisión del derecho a la privacidad, a través de dar a conocer datos sensibles, frente a la seguridad pública.

Ante lo ya expuesto, a continuación se presenta un análisis de la sentencia 32/2012 de la Suprema Corte de Justicia de la Nación (SCJN), en la cual se planteó la colisión de los principios de seguridad vs. privacidad, derivado de la acción de inconstitucionalidad que impugnó lo relativo al uso de sistemas de geolocalización por parte de los ministerios públicos como una herramienta para el combate a la delincuencia. Una vez expuestos los puntos primordiales de la sentencia, se expone lo referente a la ponderación como método de resolución de coalición de principios, siguiendo con las propuestas de Robert Alexy, Guastini y Moreso; se finaliza con el análisis de la ponderación realizada por la ministra instructora ${ }^{2}$ de la sentencia 32/2012.

\section{Síntesis DE LA SENTENCIA 32/2012}

El 17 de abril de 2012 se publicó en el Diario Oficial de la Federación $(D O F)$ el decreto que aprobó la reforma a diversas disposiciones de la Ley Federal de Telecomunicaciones (LFT) y al Código Federal de Procedimientos Penales (CFPP), ambos actualmente abrogados. En su conjunto, las reformas se conocieron de forma coloquial como Ley de Geolocalización, la cual permitía la geolocalización en tiempo real en el marco de la

\footnotetext{
2 Margarita Beatriz Luna Ramos, ministra en retiro.
} 
persecución de hechos delictivos señalados en la normatividad. El legislador señala en el dictamen:

...aprobar las reformas se fortalecen las herramientas de la autoridad ministerial en el combate de delitos en materia de delincuencia organizada, secuestro, extorsión o amenazas; en específico, por lo que hace a la geolocalización. Se busca consolidar un marco legal que permitiera al Estado Mexicano investigar eficazmente, en tiempo real, los delitos en materia de delincuencia organizada, secuestro, extorsión o amenazas, para fijar geográficamente el sitio aproximado del lugar de donde se realiza la llamada proveniente de sus autores o copartícipes, a fin de aprehenderlos y, lo más importante, localizar y rescatar con vida a la víctima del secuestro, cuando se trate de ese delito (Gaceta Parlamentaria, 2012).

La iniciativa no fue bien recibida por diversos grupos sociales (Barajas, 2014), y eventualmente la Comisión Nacional de Derechos Humanos (CNDH) presentó una acción de inconstitucionalidad en relación con los artículos 133 quáter $^{3}$ del CFPP y 40 bis $^{4}$ de la LFT, al considerar que violan el derecho humano a la privacidad y/o a la vida privada, sustentados en los artículos 14 y 16 de la Constitución Política de los Estados Unidos Mexicanos, el artículo 11 de la Convención Americana sobre Derechos Humanos, el artículo 17 del Pacto Internacional de Derechos Civiles y Po-

3 "Artículo 133 Quáter: Tratándose de investigaciones en materia de delincuencia organizada, delitos contra la salud, secuestro, extorsión o amenazas, el Procurador General de la República o los servidores públicos en quienes delegue la facultad, solicitarán por simple oficio o medios electrónicos a los concesionarios o permisionarios del servicio de telecomunicaciones la localización geográfica, en tiempo real, de los equipos de comunicación móvil asociados a una línea, que se encuentren relacionados. De todas las solicitudes, la autoridad dejará constancia en autos y las mantendrá en sigilo.

En ningún caso podrá desentenderse la solicitud y toda omisión imputable al concesionario o permisionarios, será sancionada en términos de lo previsto por el artículo 178 Bis del Código Penal Federal. Se castigará a la autoridad investigadora que utilice los datos e información obtenidos como resultado de localización geográfica de equipos de comunicación móvil para fines distintos a los señalados en este artículo, en términos de lo establecido en la fracción IV del artículo 214 del Código Penal Federal".

4 "Artículo 40 Bis: Los concesionarios o permisionarios del servicio de telecomunicaciones, están obligados a colaborar con las autoridades en la localización geográfica, en tiempo real, de los equipos de comunicación móvil asociados a una línea que se encuentren relacionados con investigaciones en materia de delincuencia organizada, delitos contra la salud, secuestro, extorsión o amenazas a solicitud del Procurador General de la República, de los procuradores de las entidades federativas o de los servidores públicos en quienes deleguen esta facultad, de conformidad con las leyes correspondientes.

Cualquier omisión o desacato a estas disposiciones será sancionada por la autoridad, en los términos de lo previsto por el artículo 178 Bis del Código Penal Federal”. 
líticos, el artículo 12 de la Declaración Universal de Derechos Humanos, y el artículo 16 de la Convención sobre los Derechos del Niño, además de ser contrarios a las garantías de legalidad y seguridad jurídica.

Los preceptos impugnados por la Comisión Nacional de Derechos Humanos se resumen en los siguientes:

1) Intervención de la autoridad judicial en la autorización, supervisión y evocación de la solicitud de localización geográfica de un equipo de comunicación móvil. Lo anterior contraviene los artículos 14, segundo párrafo, ${ }^{5}$ y 16, primer párrafo, ${ }^{6}$ de la Constitución, ya que merma el derecho a la privacidad de una persona al no existir una orden de autoridad judicial que fundada y motivadamente autorice la medida, supervise su aplicación, y la revoque en un tiempo determinado; contrario a lo que ocurre con la intervención de comunicaciones o el cateo.

2) La falta de precisión de los sujetos destinatarios de la medida, ya que el artículo 40 bis de la LFT no determina a quiénes se dirige la norma, sino que sólo refiere "equipos de comunicación móvil asociados a una línea, que se encuentren relacionados con investigaciones en materia de delincuencia organizada, delitos contra la salud, secuestro, extorsión o amenazas", con lo que permite su aplicación a quienes sus equipos móviles estén relacionados o asociados con el investigado; de ese modo, un tercero no sospechoso en la investigación también podría ser sujeto de vigilancia.

3) Límite temporal. Toda medida gubernamental que violente los derechos humanos debe regular sus alcances y límites, como es el temporal, de manera clara y específica, lo cual no se advierte en las normas impugnadas.

La CNDH concluyó que la ausencia de estos elementos impide que la norma cumpla con los requisitos de legalidad, certeza y seguridad jurídica que exige la Constitución mexicana, haciéndola una disposición abierta con un gran potencial para transgredir derechos fundamentales; además,

5 "Artículo 14... Nadie podrá ser privado de la libertad o de sus propiedades, posesiones o derechos, sino mediante juicio seguido ante los tribunales previamente establecidos, en el que se cumplan las formalidades esenciales del procedimiento y conforme a las Leyes expedidas con anterioridad al hecho...”.

"Artículo 16. Nadie puede ser molestado en su persona, familia, domicilio, papeles o posesiones, sino en virtud de mandamiento escrito de la autoridad competente, que funde y motive la causa legal del procedimiento...”. 
no es proporcional, ya que aun cuando persigue un fin legítimo, como el que el Estado cuente con mejores y mayores herramientas para combatir el crimen organizado y brindar justicia a las víctimas, para ello vulnera los derechos de certeza, seguridad jurídica, legalidad y privacidad (Ochoa, 2015: 45).

Conceptos y derechos analizados en la sentencia

\section{A. Geolocalización}

Está definida en el artículo 3o. de la LFT como la localización geográfica en tiempo real, así como la ubicación aproximada en el momento en que se procesa una búsqueda de un equipo terminal móvil asociado a una línea telefónica determinada. Esta herramienta deviene de la interacción con las nuevas tecnologías: se considera una herramienta al servicio de las personas, pero cabe cuestionar hasta qué punto esta herramienta debe ser usada por las instituciones estatales para combatir delitos y establecer un bien común.

\section{B. Derecho a la privacidad}

El derecho a la privacidad o intimidad se prevé en el primer párrafo del artículo 16 de la Constitución, que establece, en términos generales, la garantía de seguridad jurídica de los gobernados a no ser molestados en la privacidad de su persona, intimidad familiar, papeles o posesiones, salvo el caso excepcional de existir mandato fundado y motivado de la autoridad competente, lo cual tiene como fin el respeto a un ámbito de vida privada personal y familiar que, por regla general, debe excluirse del conocimiento ajeno y de las intromisiones de los demás.

El derecho a la privacidad da la facultad a todo individuo de separar aspectos de su vida privada del escrutinio público, como lo señala García (s. a.). Siguiendo a este autor, se reconoce que este derecho tiene dos componentes: el primero es el derecho a aislarse, y el segundo es el derecho a controlar la información de uno mismo a pesar de haberla divulgado con anterioridad. Helen Nissenbaum apunta que el valor de la privacidad está en su relación funcional con fines valorados como el bienestar y desarrollo humanos: la creatividad, la autonomía, la salud mental y la libertad (Nissenbaum, 2011: 85). 
El espectro de protección que abarca el derecho a la privacidad incluye todos aquellos aspectos que influyen en el desarrollo personal. Este derecho se ve constantemente vulnerado ante los avances tecnológicos, ya que hay mayores posibilidades de que exista una intromisión a la privacidad sin ser conscientes de ello. Al igual que otros derechos fundamentales, el derecho a la privacidad no es absoluto y es dinámico; cuando éste se ve enfrentado con otro derecho fundamental, un análisis ponderado resulta útil para dar solución a un caso concreto.

\section{Ponderación COMO TÉCniCA de SOluCión DE CONFLICTO DE PRINCIPIOS}

En palabras de Bernal (1989: 6), se señala que la ponderación es la forma en cómo se aplican los principios jurídicos, es decir, las normas que tienen la estructura de mandatos de optimización. Siguiendo a Robert Alexy, estas normas no determinan exactamente lo que debe hacerse, sino que ordenan "que algo sea realizado en la mayor medida posible, dentro de las posibilidades jurídicas y reales existentes" (Alexy, 2008: 83). Las posibilidades jurídicas están determinadas por los principios y reglas opuestas, y las posibilidades reales se derivan de enunciados fácticos.

La ponderación es hoy en día un criterio metodológico primordial en el ejercicio judicial. Los tribunales constitucionales, tribunales superiores y demás similares, encargados de analizar las normas constitucionales de cada país y/o aplicación de tratados internacionales en materia de derechos fundamentales, han prestado suma atención a las discusiones teóricas de aplicación de la ponderación a través del análisis de la estructura y de los límites de los principios. Sin embargo, la ponderación se sitúa en el centro de muchas discusiones teóricas que revelan que algunos aspectos, tales como su estructura y sus límites, distan de estar del todo claros, y existen diferentes propuestas de modelos de ponderación (Sardo, 2013: 239-272).

En América Latina, los principales defensores de la ponderación son aquellos autores llamados neo-constitucionalistas, y que consideran:

1) A los sistemas jurídicos como conjuntos de dos tipos de normas: reglas y principios (tesis principialista).

2) Al derecho como un fenómeno vinculado con la moral en varios niveles: definitorio, conceptual y de justificación (tesis de la conexión necesaria). 
3) Al razonamiento jurídico como parte de la racionalidad práctica general (tesis del caso especial).

\section{1. ¿Qué es un principio?}

Los principios son un mandato de optimización. Son normas que ordenan que algo sea realizado en la mayor medida posible, de acuerdo con las posibilidades fácticas y jurídicas. Autores como Carrió y Gastini (citados en Atienza y Ruiz, 1996: 3-25) enumeran seis sentidos significativos de un principio:

1) Principio en el sentido de norma muy general, entendiendo por tal la que regula un caso cuyas propiedades relevantes son muy generales.

2) Principio en el sentido de norma redactada en términos particularmente vagos, gracias a la presencia de conceptos jurídicos indeterminados o dúctiles, como orden público, abuso de derecho, etcétera.

3) Principio como norma programática o directriz, esto es, de norma que dispone la obligación de perseguir determinados fines.

4) Principio como norma que expresa valores superiores de un ordenamiento jurídico y que son el reflejo de una determinada forma de vida, de un sector del mismo, o de una institución.

5) Principio como norma dirigida a los órganos de aplicación del derecho y que señala, con carácter general, cómo se debe seleccionar la norma aplicable, interpretarla, etcétera.

6) Principio como regula iuris, esto es, de enunciado o máxima de la ciencia jurídica de un considerable grado de generalidad y que permite la sistematización del ordenamiento jurídico o de un sector del mismo. Tales principios pueden o no estar incorporados al derecho positivo.

\section{Modelo de ponderación de Riccardo Guastini}

En el análisis que realiza Guastini sobre la ponderación se señala que una teoría completa de los conflictos entre principios constitucionales debería incluir: a) una tipología de los conflictos entre normas; $b$ ) un análisis de la estructura normativa de los principios, y c) un análisis de los procedi- 
mientos interpretativos (en sentido amplio) utilizados para la solución de los conflictos entre principios (Guastini, 2007: 631).

Guastini parte de las diferencias entre reglas y principios constitucionales. Las reglas las define como enunciados condicionales que conceden una consecuencia jurídica a una clase de hechos. De los principios señala y destaca sus características de fundamentales (que brindan un sustento axiológico y ético-político al sistema jurídico) e indeterminables (que la distinguen como defectibles y genéricas). Siguiendo al mismo autor, la manera de solucionar los conflictos entre derechos fundamentales parte del análisis de los conflictos entre principios constitucionales y el empleo de la técnica de la ponderación (2007: 631).

Sobre la tipología de los conflictos normativos, distingue entre dos tipos de conflictos: 1) en abstracto / necesarios, y 2) en concreto / contingentes. Sobre los del segundo tipo hace un análisis más detallado, ya que habitualmente se encuentran éstos y no los del primer tipo. Señala que un conflicto en concreto es el que se produce cada vez que - al momento de la aplicación del derecho en un caso concreto- se observa que dos normas conceden dos consecuencias jurídicas incompatibles al mismo caso concreto (Guastini, 2007: 631). Siguiendo lo señalado sobre los conflictos concretos, éstos dependen de lo que ocurre en el mundo, en tanto que los conflictos abstractos dependen de la estructura del lenguaje legislativo. Se puede distinguir entre conflictos totales y parciales, lo cual es útil para su solución.

Guastini apunta que los principios son normas fundamentales e indeterminadas que se distinguen de las reglas, ya que "se puede convenir que una regla sea un enunciado condicional que concede determinada consecuencia jurídica a una clase de hechos" (2007: 631). Un principio es una norma fundamental; es decir, una norma que, en primer lugar, caracteriza el sistema jurídico del cual trata (o de uno de sus sectores), en el sentido de que constituye un elemento esencial para la identificación de la fisonomía del sistema; en segundo lugar, da fundamento axiológico (otorga justificación ético política) a una pluralidad de otras normas del sistema, y en tercer lugar, no exige a su vez algún fundamento o alguna justificación ético política porque es concebida, en la cultura jurídica existente, como una norma evidentemente "justa" o "correcta". Un principio es una norma indeterminada; habla de una indeterminación peculiar que puede asumir dos formas distintas: la defectibilidad (defeasibility) y la generalidad.

El autor señala que los principios como normas defectibles no establecen exhaustivamente los hechos condicionantes; es decir, no enumera todas las excepciones; mientras que como normas genéricas exigen, por un 
lado, la formulación de otra norma que lo concretice y permita su actuación o ejecución ( por otro lado, que pueda ser actuada enseguida o concretizada en muchos modos diversos y alternativos (Guastini, 2007: 631).

Con la ponderación se establece una jerarquía axiológica móvil entre dos principios en conflicto, los cuales tienen las siguientes características:

1) En primer lugar, se trata de un conflicto entre normas que, de ordinario, emanan en el mismo momento.

2) En segundo lugar, se trata de un conflicto entre normas que tienen el mismo estatuto formal, la misma posición en la jerarquía de las fuentes del derecho.

3) En tercer lugar, se trata de un conflicto "en concreto".

La jerarquía axiológica es una relación de valores creada por el juez constitucional mediante un juicio comparativo de valores. Es un enunciado dotado de forma lógica, lo cual supone atribuir a uno de los principios una importancia mayor respecto al otro. Guastini señala que la ponderación no es un conciliación, ya que no consiste en poner de acuerdo los dos principios en conflicto ni en encontrar un punto de equilibrio entre ellos; tampoco consiste en la aplicación o en el sacrificio parcial de dos principios (Guastini, 2007: 631). La ponderación ayuda a resolver cuál de los dos principios es aplicado, y consecuentemente acotado. Sobre la característica de móvil, apunta que es una jerarquía que vale para el caso concreto, pero que podría ser revertida - y que a menudo es revertidaen un caso concreto diverso.

La ponderación trae aparejado un ejercicio discrecional del juez, el cual Guastini señala que es doblemente discrecional. En primer lugar, al crear una jerarquía axiológica entre los principios, y, en segundo lugar, derivado de la mutación de valores comparativos de los principios ante una nueva controversia (Guastini, 2014: 78).

\section{Modelo de ponderación de Moreso}

José Juan Moreso (citado en Sardo, 2013: 239-272) propone un modelo de la ponderación que, por un lado, se apoya en la teoría de los sistemas normativos de Alchourrón y Bulygin, y por el otro, defiende una forma de objetivismo ecuménico que parece encontrar su justificación fundamental en una forma de intuicionismo perceptivo asociada a un cierto tipo de 
constructivismo moral. Para Moreso (2006), los conflictos sólo son aparentes y pueden ser resueltos de manera correcta con un refinamiento de los sistemas de normas constitucionales, especificando sus contenidos y buscando coherencia en el sistema. Esta teoría asume las siguientes tesis:

1) Las normas (o principios) constitucionales tienen una doble dimensión: moral y jurídica.

2) Cuando parece que hay un conflicto entre principios se puede utilizar la ponderación.

3) La mejor manera de concebir la ponderación es como un proceso de especificación.

4) La especificación, a su vez, puede ser (re)construida como una operación lógica de revisión del antecedente de las normas constitucionales, introduciendo nuevas propiedades relevantes que puedan ser identificadas de manera objetiva.

5) La objetividad está garantizada por nuestras intuiciones morales básicas compartidas y por la presencia de casos paradigmáticamente justos o injustos, que constituyen el núcleo de los derechos fundamentales.

Para Moreso, es posible concebir la ponderación como un proceso racional (tiene una estructura lógica) que permite una distribución coherente y armoniosa de los principios/valores constitucionales en el sistema, además que puede ofrecer respuestas moral y jurídicamente correctas. Para el autor, es mejor pensar en un modo de configurar la ponderación como un paso previo a la subsunción: una vía según la cual la ponderación es la operación que permite pasar de las normas que establecen derechos fundamentales (que tienen la estructura de principios y pautas) a las condiciones de aplicación abiertas de la subsunción en el ámbito de un problema (Moreso, 2016: 18).

\section{Modelo de ponderación de Robert Alexy}

Para Robert Alexy, la ponderación es objeto del tercer subprincipio del principio de proporcionalidad que trata de la optimización relativa a las posibilidades jurídicas. El objeto de los dos primeros subprincipios (idoneidad y necesidad) es la optimización relativa de las posibilidades fácticas. Lo que se trata de evitar frente a una situación fáctica de conflicto son los costos que pueden recaer sobre los derechos fundamentales y los fines del 
legislador. La ponderación se realiza por intermedio de la llamada eficiencia de Pareto (Alexy, 2016: 2).

Alexy establece a la ponderación como una ley, la cual se divide en tres etapas:

1) Determinar el grado de no satisfacción o restricción de un principio.

2) Determinar la importancia de la satisfacción del principio contrario.

3) Determinar si la importancia del principio contrario justifica la no satisfacción o restricción del otro principio.

Las tres etapas implican juicios racionales. Primero, la intensidad; segundo, la importancia de las razones que justifican la interferencia, y tercero, la relación entre ambas. Para Alexy es importante demostrar la posibilidad de efectuar juicios racionales donde el juez pueda determinar el grado de importancia del derecho fundamental que se debe optimizar.

Para poder demostrar la racionalidad de la ponderación, Alexy señala que es necesario que se tenga en cuenta también que la ponderación se encuentra implícita en un sistema de inferencias, el cual a su vez está intrínsecamente conectado al concepto de corrección. En tanto que un sistema de inferencias, la subsunción es una regla que puede expresarse mediante un esquema deductivo llamado justificación interna, el cual se construye con la ayuda de las lógicas proposicional, de predicados y deóntica. Por lo tanto, es primordial para la teoría del discurso jurídico que en el caso de la ponderación de principios exista una contraparte de este esquema deductivo. Este esquema podría llamarse fórmula del peso (Alexy, 2016: 5). La representación más sencilla de esta fórmula es:

$$
W i, j=\frac{I i}{I j}
$$

Donde ( I i) equivale a la intensidad de la interferencia con el principio (P i); (I j) representa la importancia de satisfacer el principio en colisión; $(\mathrm{W} i, \mathrm{j})$ equivale al peso concreto de (P i). Haciendo que el peso específico sea un cociente de la intensidad de la interferencia ( $\mathrm{P}$ i) y de la importancia concreta del principio en colisión $(\mathrm{P} \mathrm{j})$, la fórmula del peso pone de manifiesto que el peso concreto de un principio es, en realidad, un peso relativo.

En tenor de lo anterior, para Alexy (2016: 8) la objeción a esta fórmula es clara, debido a que sólo se puede hablar de cocientes en la presencia de números, y en la medida en que los ejercicios de ponderación llevados 
a cabo en el derecho constitucional no utilizan números. La réplica a esta objeción podría comenzar con la observación de que el lenguaje de la lógica utilizado para demostrar la estructura de la subsunción no es utilizado en los razonamientos judiciales, y, sin embargo, que este lenguaje es la mejor vía posible para hacer explícita la estructura inferencial de las reglas. Esto mismo es aplicable para demostrar la estructura ilativa de los principios a través de números que son sustituidos por las variables de la fórmula del peso. Los tres valores del modelo tríadico (leve, moderado y grave) pueden ser representados por $l, m \mathrm{y} g$. Existen numerosas posibilidades para asignar valores a $l, m$ y $g$. Una posibilidad muy simple, pero a la vez sumamente instructiva, consiste en tomar la secuencia geométrica $2^{0}, 2^{1}$ y $2^{2}$; esto es, 1 , 2 y 4 . Sobre esta base, $l$ tiene el valor $1, m$ el valor 2 , y $g$ el valor 4 .

La racionalidad de una estructura inferencial depende esencialmente de si conecta premisas que a su vez puedan ser justificadas racionalmente. Alexy señala que las decisiones o proposiciones conectadas por la fórmula del peso deben ser justificadas mediante ulteriores argumentos.

La fórmula del peso es una clase de argumento. Las formas de argumento definen la estructura lógica de movimientos dentro de los discursos y muestran cómo los movimientos se relacionan entre sí. La ponderación está conectada con el discurso. La ponderación no es posible sin el discurso y las distintas clases de argumentos o estructuras de inferencia incluyen necesariamente la estructura ponderativa. Para Alexy, la ponderación es tan racional como el propio discurso (Alexy, 2016: 12).

\section{Ponderación en la sentencia de la Acción DE INCONSTITUCIONALIDAD 32/2012}

La sentencia de la Acción de Inconstitucionalidad 32/2012 presenta la colisión de principios con base en el derecho a la privacidad y a la seguridad en el marco del combate a la delincuencia por parte de las instituciones gubernamentales y del concepto del orden público. Puesto que el orden social es el que permite su vigencia efectiva, debe conciliarse el ejercicio de un derecho de forma que éste no impida o bloquee otros. Se precisó que dicha medida sólo procede cuando los equipos móviles asociados a una línea estén relacionados con las investigaciones de delincuencia organizada, delitos contra la salud, secuestro, extorsión o amenazas, sin que se trate de la intervención a las comunicaciones o del registro de llamadas que se realicen en esos equipos, por lo que no se vulnera el derecho a la privacidad de las personas. 
En relación con el derecho a la privacidad, la ministra instructora señaló que la posible restricción a la vida privada de una persona puede darse al solicitar la localización de un equipo de comunicación móvil, que debe ceder en interés de preservar el orden público y la paz social, garantizar la protección de los mencionados derechos y la eficaz investigación de los delitos. Además, señala que esta facultad está acotada a la ubicación del lugar de donde se realiza la llamada mediante un equipo móvil asociado a una línea, por lo que no comprende la intervención de comunicaciones ni el registro de llamadas (para las cuales existe otra reglamentación), y determina que de ahí no se pueda considerar que se vulnere el derecho a la privacidad.

Se señaló que el ejercicio de la facultad para la geolocalización está previsto para los delitos relacionados con delincuencia organizada, definida constitucionalmente en el artículo 16 como la organización de hecho de tres o más personas para cometer delitos en forma permanente o reiterada, en los términos de la ley de la materia. En esta sentencia, la SCJN puso de manifiesto que la geolocalización de un equipo permitiría la detección de otros equipos asociados con la misma línea y, en su caso, de los copartícipes organizados para delinquir. Por ello, para la SCJN el combate a la delincuencia organizada, contenida en el principio de seguridad pública, es de mayor relevancia que la protección del derecho a la privacidad, en tanto que la autoridad ministerial intervenga comunicaciones o realice el cateo del lugar, cumpla con las formalidades previstas en las leyes aplicables.

Así, la ministra instructora concluyó que el mencionado artículo 133 no vulnera los derechos fundamentales que aduce la CNDH, ni aun cuando de la investigación derive la localización de una persona, pues el objeto de dicha investigación a cargo del Ministerio Público es practicar y ordenar la realización de todos los actos para acreditar el cuerpo del delito y la probable responsabilidad del inculpado, para lo cual puede valerse de medios tradicionales (como son los testigos y las huellas digitales) o de aquellos que son producto de la innovación tecnológica, entre ellos los sistemas de localización satelital.

\section{Conclusión}

La geolocalización, conforme al marco normativo en México, se considera lícita, siempre y cuando sea solicitada por el procurador general de la República, por los procuradores de las entidades federativas o por los servidores 
públicos en quienes legalmente se delegue esta facultad, y cuando la misma sea solicitada únicamente para la realización de investigaciones en materia de delincuencia organizada, delitos contra la salud, secuestro, extorsión o amenazas.

Sin embargo, las autoridades que hagan uso de este tipo herramientas, mecanismos y procedimientos que se desprendan de la ciencia y tecnología, deben observar los principios contenidos en la Constitución Política de los Estados Unidos Mexicanos para evitar atropellos y la vulneración de algunos derechos so pretexto de la protección de otros. En tanto, el uso de la geolocalización debe ser ponderada, considerando la colisión que se podría generar entre la seguridad, integridad física y el bien común con el derecho a la privacidad y la protección de los datos personales (Martínez, 2016).

Ante la resolución, se escucharon voces contrarias. Alegando la potencial vulneración del derecho a la privacidad de las personas, la entonces comisionada del Instituto Nacional de Acceso a la Información y Protección de Datos Personales (INAI), Areli Cano, señaló que la localización geográfica en tiempo real de equipos de comunicación móvil es una medida de vigilancia encubierta que se lleva a cabo sin el consentimiento del titular de los datos personales; es decir, se realiza en secreto, por lo que merece un tratamiento jurídico acorde a su naturaleza de investigación para inhibir los abusos y arbitrariedades que conllevan este tipo de medidas (INAI, 2014: 3-14).

La SCJN, en su sentencia, señala que la geolocalización es lícita y considerada constitucional ante situaciones excepcionales; sin embargo, en ninguna de las normas analizadas en dicha sentencia se señalan cuáles son éstas, dando la posibilidad a un uso arbitrario por parte de las autoridades ministeriales.

\section{BibLiografía}

Alexy, Robert, 2016, Jueces y ponderación argumentativa, México, UNAM, Instituto de Investigaciones Jurídicas.

ALEXY, Robert, 2008, El derecho y la naturaleza del derecho, España, Marcial Pons.

Atienza, Manuel y Ruiz, Juan, 1996, Las piezas del derecho. Teoría de los enunciados jurídicos, España, Ariel Derecho. 
Barajas, Fernando, 2014, “¿Por qué debe preocuparnos la Ley de Geolocalización?”, Revista Digital Código Espagueti, 14 de enero, disponible en: https:/codigoespagueti.com/internet/ley-de-geolocalizacion-mexico/.

Bernal, Carlos, 1989, "La estructura y los límites de la ponderación”, en Cuadernos de Filosofía del Derecho (DOXA), España, Universidad de Alicante.

148 Estrada-VÉlez, Sergio, 2010, "La ponderación o la débil frontera entre la arbitrariedad y el uso legítimo de la discrecionalidad", Revista Vniversitas. Revista de Ciencias Jurídicas, núm. 121, julio-diciembre.

- Gaceta Parlamentaria, 2012, "Dictamen de las Comisiones Unidas de Justicia, de Comunicaciones, y de Seguridad Pública, con proyecto de decreto que reforma, adiciona y deroga diversas disposiciones de los Códigos Federal de Procedimientos Penales, y Penal Federal, así como de las leyes Federal de Telecomunicaciones, que establece las Normas Mínimas sobre Readaptación Social de Sentenciados, y General del Sistema Nacional de Seguridad Pública”, año XV, núm. 3455-II, martes 21 de febrero de 2012, disponible en: http://gaceta.diputados.gob.mx/.

García, Diego, s. a., Derecho a la privacidad, México, Tríptico de la Comisión Nacional de Derechos Humanos.

Guastini, Ricardo, 2014, Estudios sobre la interpretación jurídica, México, UNAM, Instituto de Investigaciones Jurídicas.

Guastini, Ricardo, 2007, "Ponderación: un análisis de los conflictos entre principios constitucionales", Palestra del Tribunal Constitucional. Revista Mensual de Jurisprudencia, Perú, año 2, núm. 08, agosto, disponible en: https://docplayer.es/84633-Ponderacion-un-analisis-de-los-conflictos-entreprincipios-constitucionales-1.html.

Instituto Nacional de Transparencia, Acceso a la Información y Protección de Datos Personales, 2014, Resuelve pleno del IFAI no presentar acción de inconstitucionalidad en contra de Ley Telecom, disponible en: http://inicio.ifai.org.mx/Comunicados/Comunicado\%20IFAI-053-14.pdf.

Martínez, Rigoberto, 2016, Geolocalización entre el bien común y el derecho a la privacidad, disponible en: http://asesoresensoluciones.com/index.php/ geolocalizacion-entre-el-bien-comun-y-el-derecho-a-la-privacidad.

Moreso, Juan, 2006, "Dos concepciones de la aplicación de las normas fundamentales", Revista Direito GV, julio-diciembre.

Nissenbaum, Helen, 2011, Privacidad amenazada, México, Océano.

OchoA, Roberto, 2015, "Geolocalización de equipos de comunicación móvil, en tiempo real, relacionados con la investigación de delitos de delin- 
cuencia organizada, contra la salud, secuestro, extorsión o amenazas", en Decisiones relevantes de la Suprema Corte de Justicia de la Nación, México, SCJN-UNAM, Instituto de Investigaciones Jurídicas, núm. 82. SARdo, Alessio, 2013, "Teorías de la ponderación. Análisis crítico", en GranDez, Pedro y Morales, Félix (eds.), La argumentación jurídica en el Estado constitucional, Lima, Palestra Editores.

SCJN, Acta de sesión pública, 15 de marzo de 2018, disponible en: https:// www.scjn.gob.mx/sites/default/files/actas-sesiones-publicas/documento/ 2018-03-23/SP-30-323125110-0001.pdf.

SCJN, Resumen de sentencias relevantes del Pleno de la Suprema Corte de Justicia de la Nación, Acción de Inconstitucionalidad 32/2012 "Valida SCJN las normas que sustentan la geolocalización de los equipos de comunicación móvil vinculados a delitos considerados graves, disponible en: http:// www2.scjn.gob.mx/AsuntosRelevantes/pagina/ResultadosPub.aspx?Pagina $=18 \&$ TotalRegistros=361\&NumParticion=1 $\&$ OrganoRadicacion=0 $\&$ Fech aResolucion $=E$ TipoAsunto $=0 \&$ NumeroExpediente $=E C A P=E$ Tema $=E$ Pro movente $=E$ ActoReclamado $=E$ Materia $=0$. 